\title{
Essential histidine pairs indicate conserved haem binding in epsilonproteobacterial cytochrome $c$ haem lyases
}

\author{
Correspondence \\ Jörg Simon \\ simon@bio.tu-darmstadt.de
}

Received 22 June 2010

Revised 23 July 2010

Accepted 9 August 2010

\author{
Melanie Kern, ${ }^{1}$ Juliane Scheithauer, ${ }^{1}$ Robert G. $\mathrm{Kranz}^{2}$ and Jörg Simon ${ }^{1}$ \\ ${ }^{1}$ Institute of Microbiology and Genetics, Department of Biology, Technische Universität Darmstadt, \\ Schnittspahnstr. 10, 64287 Darmstadt, Germany \\ ${ }^{2}$ Department of Biology, Washington University, 1 Brookings Drive, St Louis, MO 63130, USA
}

\begin{abstract}
Bacterial cytochrome $c$ maturation occurs at the outside of the cytoplasmic membrane, requires transport of haem $b$ across the membrane, and depends on membrane-bound cytochrome $c$ haem lyase (CCHL), an enzyme that catalyses covalent attachment of haem $b$ to apocytochrome c. Epsilonproteobacteria such as Wolinella succinogenes use the cytochrome $c$ biogenesis system II and contain unusually large CCHL proteins of about 900 amino acid residues that appear to be fusions of the $\mathrm{CcsB}$ and $\mathrm{Ccs} A$ proteins found in other bacteria. CcsBA-type CCHLs have been proposed to act as haem transporters that contain two haem $b$ coordination sites located at different sides of the membrane and formed by histidine pairs. W. succinogenes cells contain three CcsBA-type CCHL isoenzymes ( $\mathrm{Nrfl}, \mathrm{Ccs} \mathrm{A} 1$ and $\mathrm{CcsA} 2$ ) that are known to differ in their specificity for apocytochromes and apparently recognize different haem $c$ binding motifs such as $\mathrm{CX}_{2} \mathrm{CH}$ (by CcsA2), $\mathrm{CX}_{2} \mathrm{CK}$ (by Nrfl) and $\mathrm{CX}_{15} \mathrm{CH}$ (by CcsA1). In this study, conserved histidine residues were individually replaced by alanine in each of the $W$. succinogenes CCHLs. Characterization of $\mathrm{Nrfl}$ and CcsA1 variants in $W$. succinogenes demonstrated that a set of four histidines is essential for maturing the dedicated multihaem cytochromes $c \operatorname{NrfA}$ and MccA, respectively. The function of $W$. succinogenes CcsA2 variants produced in Escherichia coli was also found to depend on each of these four conserved histidine residues. The presence of imidazole in the growth medium of both $W$. succinogenes and $E$. coli rescued the cytochrome $c$ biogenesis activity of most histidine variants, albeit to different extents, thereby implying the presence of two functionally distinct histidine pairs in each $\mathrm{CCHL}$. The data support a model in which two conserved haem $b$ binding sites are involved in haem transport catalysed by CcsBAtype CCHLs.
\end{abstract}

\section{INTRODUCTION}

Bacteria employ at least two different maturation systems to synthesize $c$-type cytochromes. These systems commonly rely on haem $b$ transport from the cytoplasm to the outside of the cytoplasmic membrane where covalent attachment of haem $b$ to a haem $c$ binding motif (HBM) of an apocytochrome $c$ takes place (see Ferguson et al., 2008; Kranz et al., 2009 for recent reviews). This last step in cytochrome $c$ biogenesis is catalysed by a membrane-bound cytochrome $c$ haem lyase (CCHL) that recognizes the HBM (usually $\mathrm{CX}_{2} \mathrm{CH}$, but other motifs such as $\mathrm{CX}_{2} \mathrm{CK}$ and $\mathrm{CX}_{15} \mathrm{CH}$ have also been described). The best-known

Abbreviations: $\mathrm{BV}$, benzyl viologen; $\mathrm{CCHL}$, cytochrome $\mathrm{c}$ haem lyase; $\mathrm{HBM}$, haem $c$ binding motif.

A supplementary figure, showing full-length alignment of selected epsilonproteobacterial $\mathrm{CCHL}$ primary structures, and a supplementary table, listing nucleotide primers used to modify $\mathrm{CCHL}$-encoding genes, are available with the online version of this paper. cytochrome $c$ maturation system is that of Escherichia coli, which is referred to as system I or the Ccm system (Richard-Fogal et al., 2009; Sanders et al., 2010). The Ccm system is present in many Gram-negative bacteria and comprises at least eight different proteins. In contrast, a maximum of four protein components arranged in system II (also known as the Ccs system) seems to be necessary to achieve cytochrome $c$ biogenesis in Epsilonproteobacteria (for example, species of the genera Helicobacter, Campylobacter and Wolinella) and in Gram-positive bacteria such as Bacillus subtilis. The CCHL in system II is most likely to be a complex of two membrane-bound proteins: CcsB (also named ResB) and CcsA (ResC) (Ahuja et al., 2009). Notably, epsilonproteobacterial genomes encode fusion proteins called CcsBA that form 10 transmembrane domains (Frawley \& Kranz, 2009). Such proteins contain an extracellular tryptophan-rich motif (designated the WWD domain, consensus sequence $\mathrm{WGX}_{2} \mathrm{WXWD}$; possibly providing a haem $b$ binding 
platform), which is also present in CcsA/ResC and in two system I proteins (CcmC and $\mathrm{CcmF}$ ) (Richard-Fogal \& Kranz, 2010). The CcsBA proteins from Helicobacter pylori and Helicobacter hepaticus are both able to restore cytochrome $c$ maturation in system I-deficient E. coli strains, indicating that CcsBA-type enzymes function as CCHLs (Feissner et al., 2006; Richard-Fogal et al., 2007; Goddard et al., 2010).

H. hepaticus CcsBA as purified from E. coli has been shown to contain reduced haem $b$, and it has been suggested that the CcsBA-type CCHL mediates haem export to the periplasmic space (Frawley \& Kranz, 2009; Merchant, 2009). According to this model, two pairs of conserved histidine residues (Table 1) are part of one cytoplasmic and one periplasmic haem binding pocket, and are essential in facilitating haem export and may also help to keep haem $b$ in the reduced state. Variants of $H$. hepaticus CcsBA in which any of the four histidines is replaced by alanine are unable to support cytochrome $c$ biogenesis, although two of them (modified at positions 2 and 4; Table 1) are still found to contain haem $b$, albeit in the oxidized state (Frawley \& Kranz, 2009). Only the two variants lacking the histidines at positions 1 and 3 (i.e. those of the putative cytoplasmic haem $b$ binding pocket) are complemented for cytochrome $c$ biogenesis by adding imidazole to the culture medium, whereas the other two histidine variants are not. Imidazole is thought to functionally mimic the histidine side chain in these CCHL variants upon occupation of the cavity formed after the replacement of histidine by alanine, thus potentially re-enabling haem $b$ coordination. This form of functional complementation is similar to that reported earlier for a myoglobin variant in the presence of imidazole (Barrick, 1994).

The Epsilonproteobacterium Wolinella succinogenes represents an extensively investigated model organism, primarily in the field of microbial energy metabolism and bioenergetics (Simon et al., 2008; Kern \& Simon, 2009a). It is a microaerobic organism that grows by various modes of anaerobic respiration, including nitrate ammonification, thereby employing periplasmic enzyme systems for nitrate reduction to nitrite (Nap system) and for ammonification of nitrite (Nrf system) (Simon et al., 2000; Simon, 2002; Kern et al., 2007; Kern \& Simon, 2008, 2009a; Simon \& Kern, 2008). The genome of $W$. succinogenes encodes 23 different mono- and multi-haem $c$-type cytochromes and matures them by using cytochrome $c$ biogenesis system II (Kern et al., 2010). Exceptionally, W. succinogenes forms three distinct CCHLs (Nrfl, CcsA1 and CcsA2; Table 1) of the CcsBA-type that have recently been shown to differ in their HBM specificity (Hartshorne et al., 2006; Kern et al., 2010). Nrfl is specifically required to attach the unique active site haem $c$ group in pentahaem cytochrome $c$ nitrite reductase (NrfA), which is bound via a $\mathrm{CX}_{2} \mathrm{CK}$ HBM (Pisa et al., 2002). CcsA1 is apparently dedicated to enabling maturation of the octahaem cytochrome $c$ MccA, which contains a special $\mathrm{CX}_{15} \mathrm{CH} \mathrm{HBM}$ in addition to seven conventional $\mathrm{CX}_{2} \mathrm{CH}$ sequences (Hartshorne et al., 2007; Kern et al., 2010). Presumably, CcsA1 is needed only for haem attachment to the special HBM of MccA. In contrast to $n r f I$ and $\operatorname{ccs} A 1$, the c $\operatorname{s} A 2$ gene cannot be deleted from the $W$. succinogenes genome, suggesting that cytochrome $c$ biogenesis is essential for cell survival (Kern et al., 2010). Most likely, CcsA2 recognizes the standard $\mathrm{CX}_{2} \mathrm{CH} \mathrm{HBM}$ and therefore corresponds to the Helicobacter CcsBA proteins discussed above. This hypothesis is supported by the fact that CcsA2 enables the $c c m$-deficient $E$. coli mutant strain RK103 to synthesize holocytochrome $c_{4}$ from Bordetella pertussis, while Nrfl and CcsA1 do not (Kern et al., 2010).

Here, we used established or newly created genetic systems to produce variants of Nrfl, CcsA1 and CcsA2 either in $W$.

Table 1. Position of conserved histidine residues in CcsBA-type CCHLs from selected Epsilonproteobacteria

A primary structure alignment is provided in Supplementary Fig. S1. Histidine residues 1 and 3 are predicted to be located at the cytoplasmic boundary of transmembrane helices 3 and 8, respectively (cytoplasmic haem $b$ binding pocket). Histidine residues 2 and 4 are thought to be in periplasmic regions near the N-terminal end of transmembrane helix 6 and the C-terminal end of transmembrane helix 9, respectively (periplasmic haem $b$ binding pocket). Histidine residues $\varepsilon 1$ and $\varepsilon 2$ are conserved only in epsilonproteobacterial CCHL enzymes. Histidine $\varepsilon 1$ is predicted to reside in transmembrane helix 5 , whereas histidine $\varepsilon 2$ is presumably located in the periplasmic loop that connects helices 5 and 6. See Fig. 3 in Frawley \& Kranz (2009) for a corresponding CcsBA topology model.

\begin{tabular}{|c|c|c|c|c|c|c|c|c|}
\hline \multirow[t]{2}{*}{ CCHL } & \multirow[t]{2}{*}{$\begin{array}{l}\text { Total number of } \\
\text { residues }\end{array}$} & \multirow[t]{2}{*}{$\begin{array}{l}\text { Number of histidine } \\
\text { residues }\end{array}$} & \multicolumn{6}{|c|}{$\begin{array}{c}\text { Designation and position of conserved histidine } \\
\text { residues }\end{array}$} \\
\hline & & & 1 & $\varepsilon 1$ & $\varepsilon 2$ & 2 & 3 & 4 \\
\hline H. hepaticus CcsBA & 936 & 18 & 83 & 678 & 691 & 761 & 858 & 897 \\
\hline H. pylori CcsBA & 936 & 20 & 86 & 679 & 692 & 760 & 857 & 896 \\
\hline W. succinogenes $\mathrm{CcsA} 2$ & 910 & 19 & 82 & 651 & 664 & 734 & 831 & 870 \\
\hline W. succinogenes $\mathrm{NrfI}$ & 902 & 30 & 78 & 641 & 654 & 724 & 821 & 860 \\
\hline W. succinogenes CcsA1 & 897 & 13 & 84 & 639 & 652 & 722 & 820 & 859 \\
\hline
\end{tabular}

${ }^{\star}$ Residues shown in bold type were substituted in this study. 
succinogenes (Nrfl, CcsA1) or in E. coli (CcsA2). Thirteen histidine variants as well as two derivatives containing modified WWD domains were characterized with respect to their cytochrome $c$ maturation capability in the presence or absence of exogenous imidazole. The present study provides the first detailed investigation, to our knowledge, of functionally distinct CcsBA-type CCHLs produced in both homologous and heterologous cellular environments.

\section{METHODS}

Growth conditions for $W$. succinogenes and $E$. coli cells. Bacterial strains used in this study are listed in Table 2. W. succinogenes cells were grown at $37^{\circ} \mathrm{C}$ either by fumarate or by nitrate respiration, as described previously (Kröger et al., 1994; Kern \& Simon, 2009b). Brain heart infusion broth $(0.5$ or $1.3 \%$, w/v) was added where appropriate. To generate anaerobic conditions, the medium was degassed and flushed several times with dinitrogen gas.
When indicated, imidazole (Serva) was added from an anaerobic stock solution [1 M, pH 7.5 (growth by nitrate respiration) or $\mathrm{pH} 8.0$ (growth by fumarate respiration)]. Antibiotics were used at the following concentrations: kanamycin, $25 \mathrm{mg} \mathrm{l}^{-1}$; chloramphenicol, $12.5 \mathrm{mg} \mathrm{l}^{-1}$.

E. coli cells were grown in LB medium at $37{ }^{\circ} \mathrm{C}$ either aerobically with shaking at 250 r.p.m. or anaerobically in rubber-sealed tubes. Antibiotics were used at the following concentrations: ampicillin,

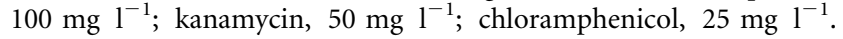
Induction of $\mathrm{Ccs} A 2$ and $\mathrm{CycC}$ production was achieved by the consecutive addition of IPTG and arabinose, as described previously (Kern et al., 2010). Imidazole (pH 7.0) was added to aerobic cultures during inoculation. Anaerobically grown cultures $(10 \mathrm{ml})$ were grown with a few modifications. After inoculation $(1 \%$, v/v, from a fresh overnight culture), the cells were incubated aerobically in the presence of imidazole for $3 \mathrm{~h}$. Subsequently, the cells were shifted to a rubbersealed tube and the oxygen content was reduced by alternate degassing and sparging with dinitrogen gas using a sterile filter. Then, IPTG $(1 \mathrm{mM})$ was added to induce $\operatorname{css} A 2$ transcription and after $2 \mathrm{~h}$, arabinose $(0.2 \%, \mathrm{w} / \mathrm{v})$ was added to induce the synthesis of

Table 2. Strains of $W$. succinogenes and $E$. coli used in this study

See Methods for details of mutant construction.

\begin{tabular}{|c|c|c|}
\hline Strain & Description and/or relevant properties ${ }^{*}$ & $\begin{array}{l}\text { Reference or } \\
\text { source }\end{array}$ \\
\hline \multicolumn{3}{|l|}{ W. succinogenes strains } \\
\hline 1. Wild-type & Type strain DSMZ 1740 & DSMZ $\dagger$ \\
\hline 2. $\Delta n r f A I J$ & Deletion mutant lacking $n r f A, n r f I$ and part of $n r f ; ; \mathrm{Km}^{\mathrm{R}}$ & Simon et al. (2000) \\
\hline 3. $\Delta n r f I J$ & Deletion mutant lacking $n r f I$ and part of $n r f ; ; \mathrm{Km}^{\mathrm{R}}$ & Simon et al. (2000) \\
\hline 4. N3 & Derivative of strain 2 containing a restored wild-type $n r f H A I J$ operon; $\mathrm{Cm}^{\mathrm{R}}, \mathrm{Km}^{\mathrm{R}}$ & Pisa et al. (2002) \\
\hline 5. NrfI H78A & Similar to strain 4 but encoding modified Nrfl $(\mathrm{H} 78 \mathrm{~A}) \ddagger ; \mathrm{Cm}^{\mathrm{R}}, \mathrm{Km}^{\mathrm{R}}$ & This work \\
\hline 6. Nrfl H641A & Similar to strain 4 but encoding modified $\operatorname{Nrfl}(\mathrm{H} 641 \mathrm{~A}) \neq ; \mathrm{Cm}^{\mathrm{R}}, \mathrm{Km}^{\mathrm{R}}$ & This work \\
\hline 7. Nrfl H724A & Similar to strain 4 but encoding modified $\operatorname{Nrfl}(\mathrm{H} 724 \mathrm{~A}) \ddagger ; \mathrm{Cm}^{\mathrm{R}}, \mathrm{Km}^{\mathrm{R}}$ & This work \\
\hline 8. NrfI H821A & Similar to strain 4 but encoding modified $\mathrm{NrfI}(\mathrm{H} 821 \mathrm{~A}) \ddagger ; \mathrm{Cm}^{\mathrm{R}}, \mathrm{Km}^{\mathrm{R}}$ & This work \\
\hline 9. $\mathrm{Nrfl} \mathrm{H} 860 \mathrm{~A}$ & Similar to strain 4 but encoding modified $\operatorname{Nrfl}(\mathrm{H} 860 \mathrm{~A}) \neq ; \mathrm{Cm}^{\mathrm{R}}, \mathrm{Km}^{\mathrm{R}}$ & This work \\
\hline 10. $\Delta m c c k a n$ & Deletion mutant lacking $m c c A, f k p A, m c c C, m c c D$ and $c c s A 1 \S ; \mathrm{Km}^{\mathrm{R}}$ & This work \\
\hline 11. $\mathrm{P}_{f r d}-m c c$ & $\begin{array}{l}\text { Derivative of strain } 10 \text { containing a restored } m c c \text { locus under the control of the } \\
\text { fumarate reductase promoter } \$ ; \mathrm{Cm}^{\mathrm{R}}\end{array}$ & This work \\
\hline 12. $\mathrm{P}_{\text {frd }}-m c c \Delta c c s A 1$ & Derivative of strain 11 lacking $\operatorname{ccs} A 1 ; \mathrm{Cm}^{\mathrm{R}}, \mathrm{Km}^{\mathrm{R}}$ & This work \\
\hline 13. CcsA1 H84A & Similar to strain 11 but encoding modified CcsAl $(\mathrm{H} 84 \mathrm{~A}) \ddagger ; \mathrm{Cm}^{\mathrm{R}}$ & This work \\
\hline 14. CcsA1 H722A & Similar to strain 11 but encoding modified CcsAl $(\mathrm{H} 722 \mathrm{~A}) \ddagger ; \mathrm{Cm}^{\mathrm{R}}$ & This work \\
\hline 15. CcsA1 H820A & Similar to strain 11 but encoding modified CcsAl $(\mathrm{H} 820 \mathrm{~A}) \ddagger ; \mathrm{Cm}^{\mathrm{R}}$ & This work \\
\hline 16. CcsA1 H859A & Similar to strain 11 but encoding modified CcsAl $(\mathrm{H} 859 \mathrm{~A}) \ddagger ; \mathrm{Cm}^{\mathrm{R}}$ & This work \\
\hline 17. CcsA1 WWD & Similar to strain 11 but encoding modified CcsAl (W799A, W801A, D802A) $\neq \mathrm{Cm}^{\mathrm{R}}$ & This work \\
\hline \multicolumn{3}{|c|}{ ( } \\
\hline $\begin{array}{l}\text { 18. RK103 pRGK332 } \\
\text { pWsCcsA2 }\end{array}$ & $\begin{array}{l}\text { Derivative of the } c c m \text { mutant strain RK103 containing two plasmids encoding } B \text {. } \\
\text { pertussis } C y c C(p R G K 332) \text { and } W . \text { succinogenes } \operatorname{Ccs} 22(\mathrm{pWsCcsA2}) ; \mathrm{Cm}^{\mathrm{R}}, \mathrm{Km}^{\mathrm{R}} ; \mathrm{Amp}^{\mathrm{R}}\end{array}$ & Kern et al. (2010) \\
\hline 19. RK103 CcsA2 H82A & Similar to strain 18 but encoding modified CcsA2 $(\mathrm{H} 82 \mathrm{~A}) \ddagger ; \mathrm{Cm}^{\mathrm{R}}, \mathrm{Km}^{\mathrm{R}} ; \mathrm{Amp}^{\mathrm{R}}$ & This work \\
\hline 20. RK103 CcsA2 H734A & Similar to strain 18 but encoding modified CcsA2 $(\mathrm{H} 734 \mathrm{~A}) \ddagger ; \mathrm{Cm}^{\mathrm{R}}, \mathrm{Km}^{\mathrm{R}} ; \mathrm{Amp}^{\mathrm{R}}$ & This work \\
\hline 21. RK103 CcsA2 H831A & Similar to strain 18 but encoding modified CcsA2 $(\mathrm{H} 831 \mathrm{~A}) \ddagger ; \mathrm{Cm}^{\mathrm{R}}, \mathrm{Km}^{\mathrm{R}} ; \mathrm{Amp}^{\mathrm{R}}$ & This work \\
\hline 22. RK103 CcsA2 H870A & Similar to strain 18 but encoding modified $\operatorname{Ccs} \mathrm{A} 2(\mathrm{H} 870 \mathrm{~A}) \neq ; \mathrm{Cm}^{\mathrm{R}}, \mathrm{Km}^{\mathrm{R}} ; \mathrm{Amp}^{\mathrm{R}}$ & This work \\
\hline 23. RK103 CcsA2 WWD & $\begin{array}{l}\text { Similar to strain } 18 \text { but encoding modified CcsA2 (W810A, W812A, D813A) } \neq ; \mathrm{Cm}^{\mathrm{R}} \text {, } \\
\mathrm{Km}^{\mathrm{R}} ; \mathrm{Amp}^{\mathrm{R}}\end{array}$ & This work \\
\hline
\end{tabular}

${ }^{*} \mathrm{Cm}^{\mathrm{R}}, \mathrm{Km}^{\mathrm{R}}$ and $\mathrm{Amp} \mathrm{p}^{\mathrm{R}}$ denote resistance to chloramphenicol, kanamycin and ampicillin, respectively.

$\dagger$ DSMZ, Deutsche Sammlung von Mikroorganismen und Zellkulturen.

$\ddagger$ See Table 1 for the position of the histidine residue within the primary structure.

$\S$ See Fig. 1. 
apo-CycC. Cells were harvested after incubation for an additional $4 \mathrm{~h}$ at $37^{\circ} \mathrm{C}$.

Cell fractionation and determination of protein concentrations. $W$. succinogenes cells harvested in the exponential or early stationary growth phase were suspended $\left(10 \mathrm{~g}\right.$ cell protein $\left.\mathrm{l}^{-1}\right)$ in an anoxic buffer ( $\mathrm{pH} 8.0$ ) containing $50 \mathrm{mM}$ Tris/ $\mathrm{HCl}$. The suspension was passed through a high-pressure cell disruption system (Constant Systems) at $135 \mathrm{MPa}$. The resulting cell homogenate was centrifuged for $15 \mathrm{~min}$ at $5000 \mathrm{~g}$ to remove cell debris. The periplasmic protein fraction of E. coli cells was obtained as described previously (Feissner et al., 2006). Protein was measured using the Biuret method with KCN (Bode et al., 1968) or the Bradford assay.

\section{Cytochrome $c$ detection and determination of specific activ-}

ities. Samples of cell proteins were subjected to SDS-PAGE using either a reducing (for $W$. succinogenes samples) or a non-reducing (for E. coli samples) loading buffer (Roth). Proteins were transferred to a PVDF membrane by Western blotting, and cytochromes $c$ were detected using the SuperSignal West Pico chemiluminescence substrate (Thermo Scientific) and exposure to X-ray film (CLXPosure film, Thermo Scientific). Nitrite reductase activity was determined by spectrophotometrically recording the rate of benzyl viologen (BV) radical oxidation by nitrite, as described previously (Kern et al., 2010). One unit of enzyme activity is defined as the oxidation of $2 \mu \mathrm{mol} \mathrm{BV} \mathrm{m^{-1 }}$. The electron transport activity from formate to nitrite was measured with intact cells of $W$. succinogenes according to the method described by Simon et al. (2001) with a few modifications. In brief, washed cells were suspended (2-5 g protein $\left.1^{-1}\right)$ in an anoxic buffer ( $\left.\mathrm{pH} 8.5\right)$ containing $150 \mathrm{mM}$ Bicine and $0.5 \mathrm{M}$ mannitol. A cell aliquot was incubated for $5 \mathrm{~min}$ at $37{ }^{\circ} \mathrm{C}$ prior to the addition of sodium formate ( $50 \mathrm{mM}$ final concentration). The reaction was started by adding $10 \mathrm{mM}$ potassium nitrite and samples were taken after various time intervals (total duration up to $30 \mathrm{~min}$ ). Electron transport activity was calculated from the nitrite production rate. One unit of electron transport activity (U) is equivalent to the consumption of $1 \mu \mathrm{mol}$ formate $\min ^{-1}$.

Construction of $\boldsymbol{W}$. succinogenes mutants. Standard genetic procedures were used (Sambrook et al., 1989). Genomic DNA was isolated from W. succinogenes using the DNeasy Tissue kit (Qiagen). PCR was carried out using Phusion High Fidelity DNA polymerase (Finnzymes) (for cloning procedures) or Biotaq Red DNA polymerase (Bioline) (for mutant and plasmid screening) with standard amplification protocols. Site-directed mutagenesis was performed using the QuikChange Site-Directed Mutagenesis kit (Stratagene) or the Phusion Site-Directed Mutagenesis kit (Finnzymes) with specifically synthesized primer pairs (Supplementary Table S1).

W. succinogenes mutants producing Nrfl variants (strains 5-9 in Table 2) were obtained from $W$. succinogenes $\Delta n r f A I J$ upon integration of pBR-N3 derivatives, resulting in a restored $n r f$ operon (Pisa et al., 2002). Site-directed mutagenesis of nrfI was performed with pBR-N3 as template and a pair of complementary primers (Supplementary Table S1). Transformation of $W$. succinogenes $\Delta n r f A I J$ with the resulting plasmids was performed by electroporation, as described previously (Simon et al., 1998). Transformants were selected in the presence of kanamycin $\left(25 \mathrm{mg} \mathrm{l}^{-1}\right)$ and chloramphenicol $(12.5 \mathrm{mg}$ $\left.1^{-1}\right)$. The desired integration of the plasmid into the genome via the $n r f H$ gene was confirmed by PCR, and each mutation was verified by sequencing an appropriate PCR product.

W. succinogenes $\Delta m c c$ kan was constructed through double homologous recombination of the wild-type genome with a deletion plasmid ( $\mathrm{p} \Delta \mathrm{mcc}$ ) designed to replace the consecutive genes $m c c A$, $f k p A, m c c C, m c c D$ and $c c s A 1$ with the kanamycin-resistance gene cartridge (kan). For homologous recombination, the respective deletion plasmid contained kan flanked by two DNA segments obtained by PCR that were identical to appropriate regions in the $W$. succinogenes genome (Fig. 1). The two PCR fragments were synthesized using the following primer pairs: 5'-GCGAATTCTTGGCATTGAGGTTGAGG-3' and 5'-CCGGATCCGTTTCCTCCTTTCAAAACAC-3' for amplifying the upstream fragment, and $5^{\prime}$ GCGGATCCAAACTCTATCTAAGGAGGTGTG-3' and 5'-CGCCATGGGATAGATCACTCCACCAAAAAAGAG-3' for the downstream fragment (black bars in Fig. 1). Primers carried EcoRI, BamHI or NcoI restriction sites (underlined) for cloning. Both fragments as well as kan (obtained by BamHI excision from pUC4K) were consecutively inserted into the high-copy-number plasmid pPR-IBA1 (IBA BioTAGnology) using appropriate restriction enzymes. PCR analysis was used to confirm that the plasmid contained kan in the same orientation as the mcc sequence. Transformants of $W$. succinogenes were selected in the presence of kanamycin $\left(25 \mathrm{mg} \mathrm{l}^{-1}\right)$. The desired deletion in the transformant genome was confirmed by PCR, and the integrity of DNA stretches involved in recombination events was confirmed by sequencing suitable PCR products.

To construct $W$. succinogenes $\mathrm{P}_{\text {frd }}$-mcc, the complete mcc gene cluster was restored on the genome of $W$. succinogenes $\Delta m c c$ kan upon integration of plasmid $\mathrm{pP}_{\mathrm{frd}}-\mathrm{mcc}$ cat (Fig. 1). This plasmid contained the deleted $m c c$ region downstream of the fumarate reductase promoter $\left(\mathrm{P}_{f r d}\right)$ and flanked by the upstream and downstream fragments of $\mathrm{p} \Delta \mathrm{mcc}$. In addition, the chloramphenicol-resistance gene cassette (cat) was inserted between the upstream fragment and the $m c c$ region. In a first step to synthesize $\mathrm{pP}_{\text {frd }}-\mathrm{mcc}$ cat, the kan gene was replaced by cat in $\mathrm{p} \Delta \mathrm{mcc}$, resulting in $\mathrm{p} \Delta \mathrm{mcc}$ cat. $\mathrm{A}_{\text {frd }}$ fragment amplified using the primer pair 5'-AAAGGGGTTTGGACGGGGTTTTG- ${ }^{\prime}$ ' and 5' $^{\prime}$-CTGTTTCCCCTGTGCAGTATTGGAGTTTC-3' was blunt end-ligated with a linear plasmid fragment obtained by PCR from $\mathrm{p} \Delta \mathrm{mcc}$ cat with the primer pair $5^{\prime}$-AAACTCTATCTAAGGAGGTGTGATGC- ${ }^{\prime}$ and $5^{\prime}$-CCGGATCCCGGTTTTTGTTAATCCGC- $3^{\prime}$, resulting in $\mathrm{pP}_{\text {frd }} \Delta \mathrm{mcc}$ cat. In a second step, the $\mathrm{f} 1$ origin of $\mathrm{pP}_{\text {frd }} \Delta \mathrm{mcc}$ cat was replaced by a SC101 origin, which lowered the plasmid number per cell (this was necessary to allow cloning of the large $m c c$ region later on). The $c a t / \mathrm{P}_{\text {frd }}$ region flanked with the upstream and downstream fragments was amplified by PCR and blunt end-ligated with the SC101 origin (obtained by BamHI/AfeI excision from pSC101 and subsequent filling in of recessed $3^{\prime}$ ends by the Klenow fragment of E. coli DNA polymerase I), resulting in $\mathrm{p}(\mathrm{lc}) \mathrm{P}_{\text {frd }} \Delta \mathrm{mcc}$ cat. In the last step of $\mathrm{pP}_{\text {frd }}$-mcc cat construction, the $m c c$ region, comprising the consecutive genes $m c c A, f k p A, m c c C$, $m c c D$ and $c c s A 1$, was inserted downstream of $\mathrm{P}_{\text {frd }}$. The $m c c$ fragment was amplified using the primer pair $5^{\prime}$-ATGAAATATTGGGACAAAGCGTTGCTGAGTC-3' and 5' -TCAAATCGTCCCCACTTTTCGGCG-3', and blunt end-ligated into a linear plasmid fragment obtained by PCR from $\mathrm{p}(\mathrm{lc}) \mathrm{P}_{\text {frd }} \Delta \mathrm{mcc}$ cat. The corresponding mutant strain $W$. succinogenes $\mathrm{P}_{\text {frd }}$-mcc was constructed by transforming W. succinogenes $\Delta$ mcc kan with $\mathrm{pP}_{\text {frd }}$-mcc cat. Transformants were selected in the presence of chloramphenicol $\left(12.5 \mathrm{mg} \mathrm{l}^{-1}\right)$, and the desired double homologous recombination was verified by PCR. The $W$. succinogenes strains producing CcsAl variants (strains 13-17 in Table 2) were obtained from $W$. succinogenes $\Delta m c c$ kan upon integration of $\mathrm{pP}_{\text {frd }}-\mathrm{mcc}$ cat derivatives containing a mutated $\operatorname{ccs} A 1$ gene. Site-directed mutagenesis of $\mathrm{pP}_{\text {frd }}$ - $\mathrm{mcc}$ cat was performed using appropriate primer pairs (Supplementary Table S1). Mutant W. succinogenes $\mathrm{P}_{\text {frd }}-m c c \Delta c c s A 1$ was obtained after transformation of $W$. succinogenes $\mathrm{P}_{\text {frd }}$-mcc with $\mathrm{p} \Delta \mathrm{ccs} \mathrm{A} 1:$ : kan (Hartshorne et al., 2007) and selection in the presence of kanamycin.

Construction of $\boldsymbol{E}$. coli mutants. Strains used in this study were derived from E. coli RK103 pRGK332 pWsCcsA2, which contains two plasmids encoding $B$. pertussis $\mathrm{CycC}$ and $W$. succinogenes CcsA2, respectively (Kern et al., 2010). To obtain plasmids encoding CcsA2 variants, $\mathrm{pWsCcsA2}$ was modified using suitable primer pairs 
W. succinogenes wild-type

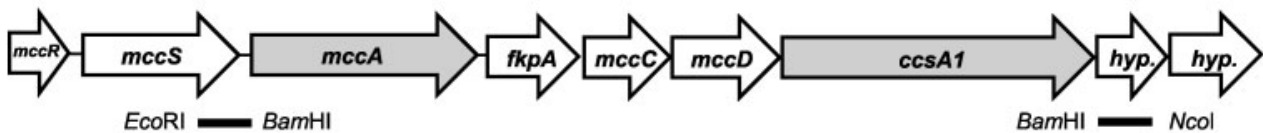

W. succinogenes $\Delta m c c$ kan
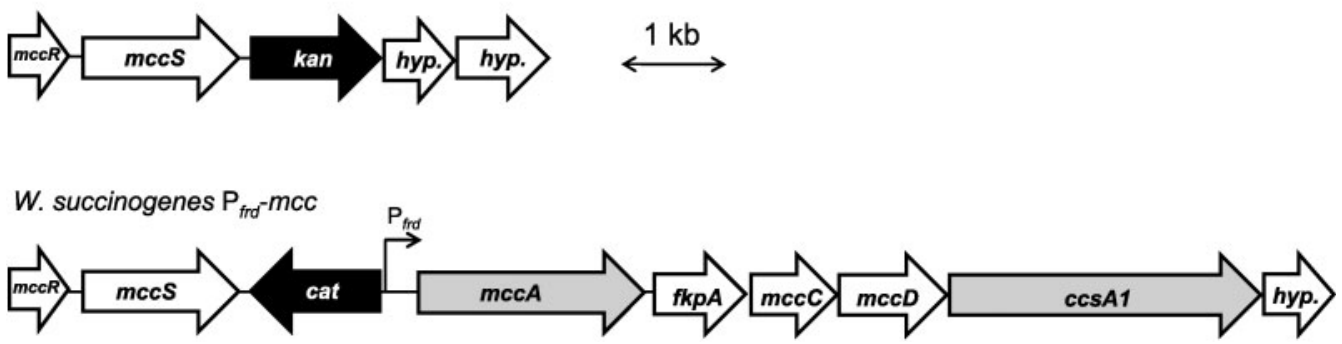

Fig. 1. Physical maps of the mcc loci in $W$. succinogenes wild-type cells and in mutant strains $\Delta m c c$ kan and $P_{\text {frd }}-m c c$. Black bars indicate regions used for homologous recombination during mutant construction (see Methods for details). $\mathrm{P}_{\text {frd }}$ and the accompanying arrow indicate the position of the fumarate reductase promoter and the corresponding transcriptional direction. The ccs $A 1$ gene encodes a CcsBA-type $\mathrm{CCHL}$ that is specifically required for the maturation of the octahaem cytochrome $c$ MccA.

(Supplementary Table S1). Transformation of E. coli RK103 pRGK332 with these plasmids resulted in strains 19-23 (Table 2).

\section{RESULTS}

\section{Production and characterization of Nrfl variants in W. succinogenes}

A previously described genetic system allowed site-directed mutagenesis of the $n r f I$ gene on a plasmid in E. coli as well as expression of the mutated gene in the context of the entire nrfHAIJ operon in W. succinogenes (Pisa et al., 2002). Here, five NrfI histidine residues (H78, H641, H724, H821 and H860; Table 1) were individually replaced by alanine and the corresponding proteins were produced in $W$. succinogenes (mutants 5-9 in Table 2). Strain W. succinogenes N3 (a control containing wild-type $n r f I$ ) and strain NrfI H641A had wild-type properties with respect to growth by both nitrate and nitrite respiration, while the other four mutants reduced nitrate to nitrite but did not grow with nitrite as electron acceptor (results not shown). These four mutants contained only very small amounts of NrfA (as judged by haem staining) and did not show any detectable nitrite reductase activity measured with reduced $\mathrm{BV}$ as artificial electron donor (Fig. 2a). Such a phenotype was also found in the $\Delta$ nrfIJ mutant (Fig. 2a) as well as in $W$. succinogenes stopI, which contained an nrfI gene inactivated by several stop codons (Pisa et al., 2002). For $W$. succinogenes stopI, it was shown that NrfA possessed only four covalently bound haem groups and lacked the active site $\mathrm{CX}_{2} \mathrm{CK}$-bound haem which is essential for nitrite reduction (Pisa et al., 2002). Therefore, it appears that the histidine residues $78,724,821$ and 860 are obligatory for Nrfl function and/or stability.
In another experiment, wild-type cells and mutants $W$. succinogenes N3, $\Delta$ nrfAIJ, $\Delta$ rffIJ, H78A, H724A, H821A and H860A were grown by nitrate respiration in the same medium as before but in the presence of exogenous imidazole (10 $\mathrm{mM}$ final concentration in the medium). Under these conditions, considerably larger amounts of NrfA were detected in the four histidine mutants along with the recovery of substantial nitrite reductase activity (between 5 and $19 \%$ relative to strain N3) (Fig. 2a). This activity was also reflected in restored electron transport activities from formate to nitrite (between 3 and $8 \%$ relative to strain N3) (Fig. 2a). Notably, the mutants could be grouped into two pairs $(\mathrm{H} 78 / \mathrm{H} 821$ and $\mathrm{H} 724 / \mathrm{H} 860$, corresponding to positions $1 / 3$ and $2 / 4$ in Table 1 ) based on similar enzyme activities and protein contents. Lowering the initial imidazole concentrations in the medium resulted in decreasing amounts of NrfA (Fig. 2b) and, apparently, the H78/H821 variants tolerated the presence of low imidazole concentrations to a greater extent than the H724/H860 variants. The addition of $10 \mathrm{mM}$ imidazole slightly impaired the growth rate of all four histidine mutants in a medium containing $50 \mathrm{mM}$ formate and $10 \mathrm{mM}$ nitrate as energy substrates but the cells reached final optical densities similar to those of control cultures grown in the absence of imidazole (results not shown). Addition of 15 or $25 \mathrm{mM}$ imidazole almost completely abolished growth of $W$. succinogenes cells during nitrate respiration.

\section{Production of CcsA1 variants in W. succinogenes cells overproducing MccA}

CcsA1 has been shown previously to be dedicated to the maturation of the octahaem cytochrome $c$ MccA, whose 

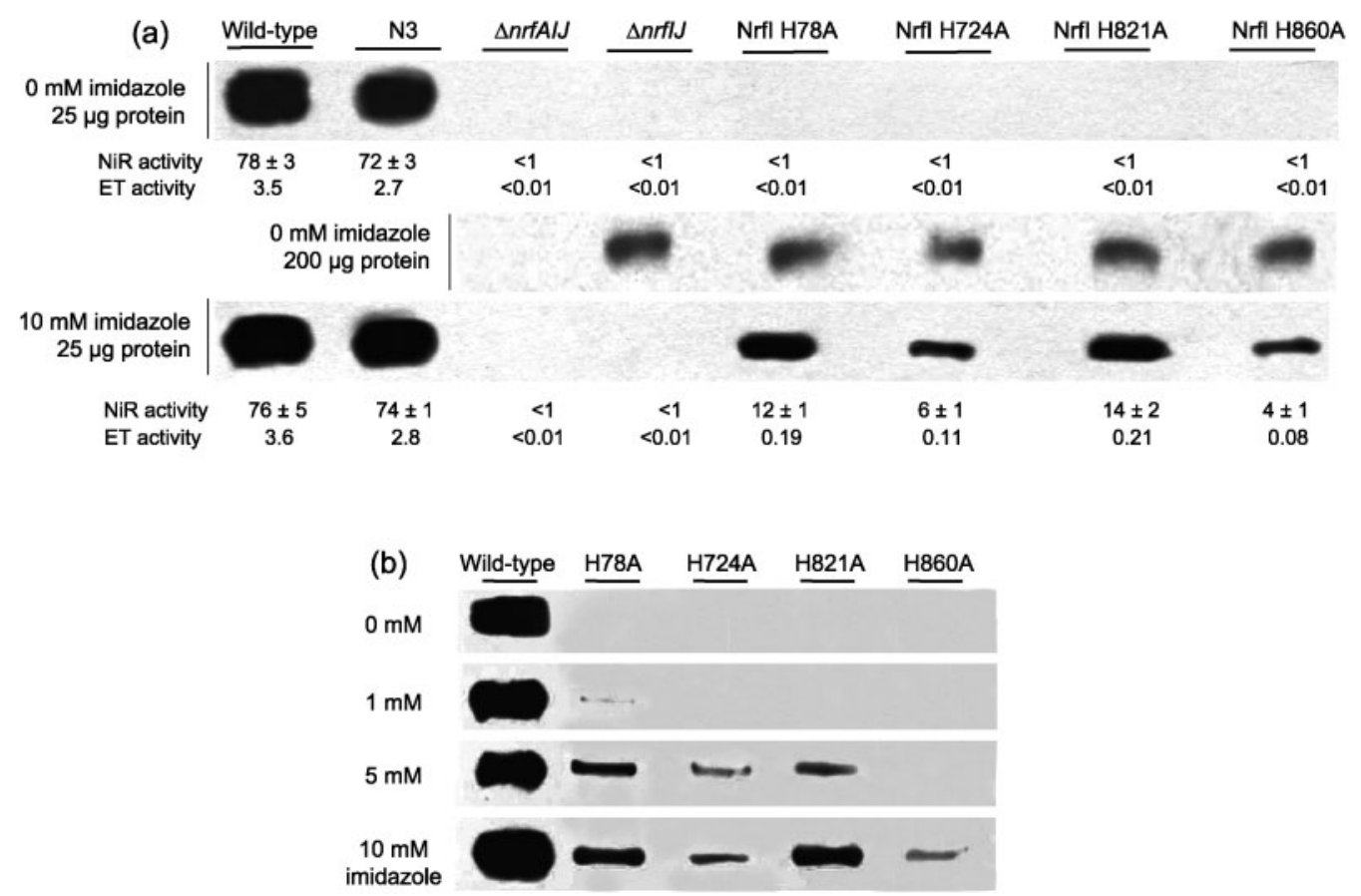

Fig. 2. Detection of cytochrome $c$ nitrite reductase (NrfA) by haem staining in cell homogenates of different $W$. succinogenes strains. Cell homogenates (protein amounts as indicated) of nitrate-grown cells were separated by SDS-PAGE and blotted onto a PVDF membrane. Only the gel region containing NrfA (at about $55 \mathrm{kDa}$ ) is shown. Numbers below the gel refer to specific activities [ $\mathrm{U}$ (mg protein $\left.)^{-1}\right]$ measured in the corresponding cell homogenate. At least three independent cultures were used for activity determination and the SD is given in the case of nitrite reductase activity, whereas representative values are shown for electron transport activities. Initial imidazole concentrations added to the cultures are shown at the left of each gel section. NiR activity, specific nitrite reductase activity; ET activity, specific electron transport activity from formate to nitrite.

function in $W$. succinogenes is not known (Hartshorne et al., 2007; Kern et al., 2010). To assess the role of single amino acid residues of CcsA1 in W. succinogenes, a genetic test system was established that allowed site-directed mutagenesis of $\operatorname{cs} A 1$ in the background of a strain whose $m c c$ gene cluster was expressed from the fumarate reductase promoter $\left(\mathrm{P}_{\text {frd }}\right)$ (Fig. 1). The same frd promoter element has been shown previously to be able to initiate expression of the mcc locus (Hartshorne et al., 2007). In a first step, a deletion mutant (W. succinogenes $\Delta m c c k a n$ ) was constructed that lacked the consecutive genes $m c c A$, $f k p A, m c c C, m c c D$ and $c c s A 1$ (Table 2). The entire $m c c A$ locus was then restored on the genome of the deletion mutant by double homologous recombination between its genome and a suitable plasmid that carried $\mathrm{P}_{\text {frd }}$ upstream of $m c c A$ (Fig. 1). The resulting strain (W. succinogenes $\mathrm{P}_{\text {frd }}$ $m c c)$ was found to produce MccA under fumaraterespiring growth conditions (Fig. 3, lane 1). The formation of MccA under these conditions was already known to depend on the presence of ccsA1 (Hartshorne et al., 2007), and therefore it was not surprising that MccA could not be detected by haem staining in cells of strain $\mathrm{P}_{f r d}-m c c$ after deletion of the ccsA1 gene (Fig. 3, lane 2).

Derivatives of $W$. succinogenes $\mathrm{P}_{\text {frd }}$-mcc were constructed that produced variants of CcsA1 (strains 13-17 in Table 2).
Each of the histidine residues H84, H722, H820 and H859 of CcsA1 was replaced by alanine, and a further CcsA1 variant was constructed that contained an AGAA sequence instead of WGWD within the WWD domain (mutant $W$. succinogenes CcsAl WWD). In the absence of exogenous imidazole, low amounts of MccA were detected in strains CcsA1 H84A and CcsA1 H820A, whereas MccA was not found in the other three mutants (Fig. 3, lanes 3-7). Increasing amounts of added imidazole ultimately resulted in the detection of MccA in all four histidine mutants, and two mutant pairs were identified whose phenotypes were apparently identical (Fig. 3). These pairs (H84/H820 and H722/H859) corresponded to CcsAl variants with modified histidines at positions $1 / 3$ and $2 / 4$, similar to the results observed for NrfI (see above). In contrast to the histidine variants, CcsAl containing the modified WWD domain was not able to produce detectable MccA, irrespective of the presence of imidazole (Fig. 3).

\section{Characterization of $\boldsymbol{W}$. succinogenes CcsA2 variants using $\mathbf{c c m}$-deficient $E$. coli RK103 cells}

As CcsA2 proved to be essential for growth of $W$. succinogenes cells (Kern et al., 2010), it was not possible to produce CcsA2 variants in a homologous system. 


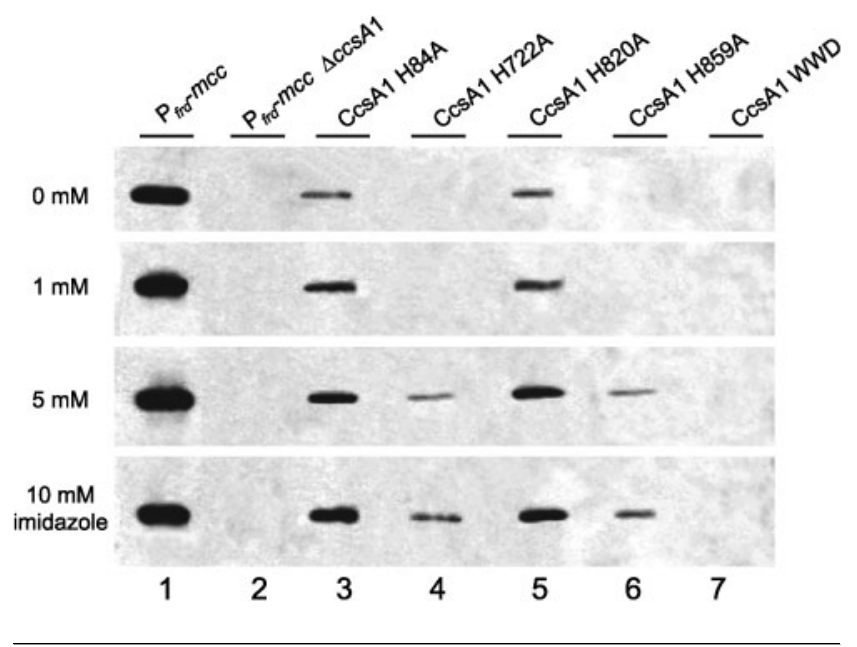

Fig. 3. Detection of MccA by haem staining in cell homogenates of different $W$. succinogenes strains. Fumarate-grown cells were separated by SDS-PAGE (100 $\mu \mathrm{g}$ protein applied per lane), blotted onto a PVDF membrane and subjected to haem staining. Only the gel region containing MccA (at about $75 \mathrm{kDa}$ ) is shown. Initial imidazole concentrations added to the cultures are shown at the left of each gel image.

Instead, $W$. succinogenes CcsA2 was synthesized using the heterologous cytochrome $c$ maturation test system, employing E. coli RK103 as host cells that also produced
B. pertussis dihaem cytochrome $c_{4}$ (CycC) as reporter protein (Feissner et al., 2006). Using this approach, wildtype CcsA2 has been shown previously to mature $\mathrm{CycC}$ (Kern et al., 2010). Here, each of the CcsA2 histidines located at the conserved positions 1-4 (Table 1) was replaced by alanine, and a WWD domain variant similar to that of CcsA1 was also constructed. Each of these variants was functionally tested using the respective E. coli RK103 cells (strains 19-23 in Table 2). A strain that produced wild-type CcsA2 served as control. Grown under either aerobic or anaerobic growth conditions, none of the five CcsA2 variants was found to be capable of $\mathrm{CycC}$ maturation, in contrast to wild-type CcsA2 (Fig. 4). When grown in the presence of imidazole, however, mutants CcsA2 H82A and CcsA2 H831A (histidine positions 1 and 3) produced stable holo-CycC, the amount of which increased with higher imidazole concentrations (Fig. 4). Notably, this effect was observed only in anaerobically grown cells. In the other three mutants, $\mathrm{CycC}$ maturation was not restored by imidazole, irrespective of the oxygen content of the cultures (Fig. 4).

\section{DISCUSSION}

Haem is synthesized in the bacterial cytoplasm and needs to be exported in order to serve as a CCHL substrate. For the bacterial cytochrome $c$ biogenesis system II, recently

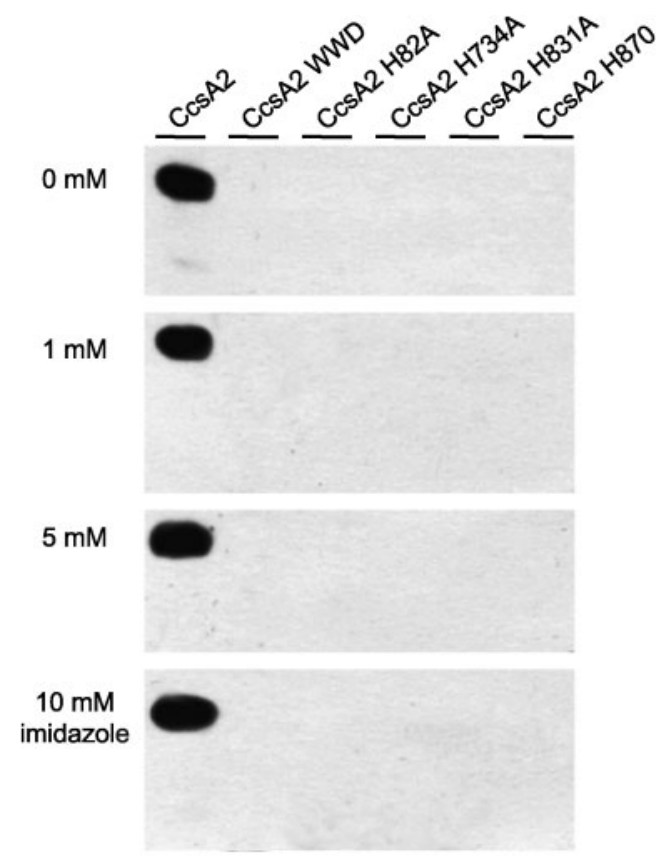

Aerobic growth

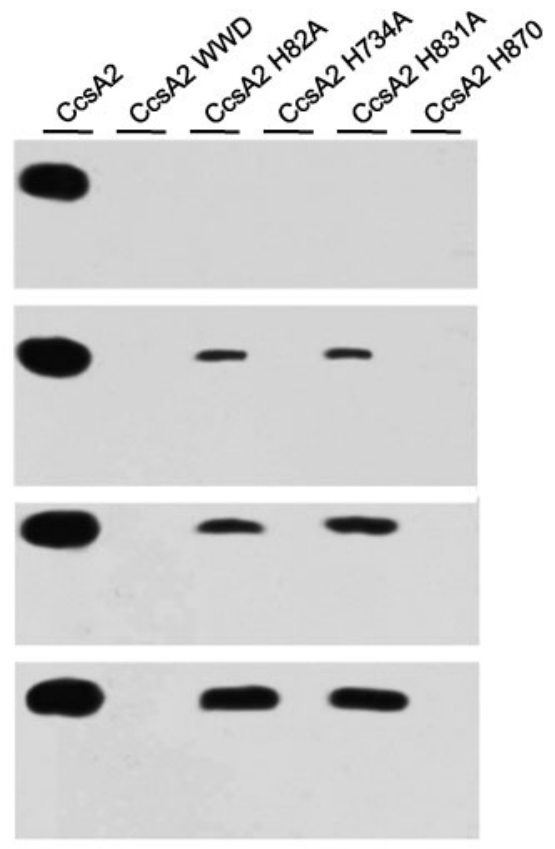

Anaerobic growth

Fig. 4. Detection of $B$. pertussis cytochrome $c_{4}(\mathrm{CycC})$ in periplasmic extracts of different $E$. coli strains. Samples from cells grown under aerobic or anaerobic conditions were separated by SDS-PAGE, blotted onto a PVDF membrane and subjected to haem staining ( $50 \mu \mathrm{g}$ protein applied per lane). Only the gel region containing cytochrome $c_{4}$ (at about $24 \mathrm{kDa}$ ) is shown. Concentrations of imidazole added to the cultures are shown at the left of the figure. 
acquired evidence suggests that CcsBA-type CCHLs mediate haem export with the help of two haem $b$ binding sites located on different sides of the membrane (Frawley \& Kranz, 2009; Kranz et al., 2009; Merchant, 2009; Goddard et al., 2010). According to this model, two pairs of histidines arranged in cytoplasmic and periplasmic haem $b$ binding pockets, respectively, serve in axial haem $b$ ligation during the export process. This hypothesis is supported by the fact that imidazole addition to the growth medium leads to restored cytochrome $c$ maturation activity of $H$. hepaticus CcsBA histidine variants when produced in E. coli RK103 (Frawley \& Kranz, 2009). However, this effect was only achieved with variants H83A and H858A, which were modified at histidine positions 1 and 3 (cytoplasmic haem $b$ binding site; Table 1). Here, we have shown that imidazole complementation can be achieved in variants of all four conserved histidine residues in two CcsBA-type CCHLs (NrfI and CcsAl) from W. succinogenes, a fact that supports the idea of separate haem $b$ binding sites. It needs to be emphasized, however, that neither histidine variant used in this study showed a cytochrome-maturing activity equivalent to that of the wild-type protein, even at the highest imidazole concentration used. The results depicted in Figs 2 and 3 suggest that the function of the cytoplasmic haem $b$ binding site can be restored more effectively by imidazole than that of the periplasmic site in both NrfI and CcsA1 from $W$. succinogenes. Likewise, the cytoplasmic haem $b$ binding site also seemed to be more accessible to imidazole complementation when CcsBA-type CCHLs (either CcsBA from $H$. hepaticus or CcsA2 from $W$. succinogenes) were produced in E. coli. Interestingly, anaerobic growth conditions apparently enhanced the imidazole complementation effect, which might be due to the fact that haem is more reduced under these conditions. Histidine residues essential for cytochrome $c$ biogenesis have also been reported for CcsB and CcsA from Chlamydomonas reinhardtii and the WWD domain-containing CcmF from E. coli (Ren et al., 2002; Dreyfuss et al., 2003; Hamel et al., 2003).

Variants of CcsBA-type CCHLs from Epsilonproteobacteria carrying modifications of the WWD domain were generally inactive in cytochrome $c$ maturation, thus demonstrating the importance of this motif. The results obtained for both CcsA1 and CcsA2 in this study were similar to those reported previously for $H$. hepaticus CcsBA and for CcsA from C. reinhardtii (Hamel et al., 2003; Frawley \& Kranz, 2009). The WWD domains of the E. coli $\mathrm{CcmC}$ and $\mathrm{CcmF}$ proteins have also been subjected to modification (Schulz et al., 2000; Ren et al., 2002; RichardFogal \& Kranz, 2010). It these cases, the exchange of one conserved tryptophan residue typically resulted in loss of cytochrome $c$ maturation activity. The molecular function of the WWD domain is not understood, but it is thought to be located near the periplasmic haem $b$ binding pocket, where it possibly plays a role in CCHL function subsequent to haem $b$ export, for example in proper presentation of haem in order to facilitate the haem lyase reaction.
Taken together, the results presented in this study suggest that CcsBA-type CCHLs from different Epsilonproteobacteria share a common architecture that comprises 10 conserved transmembrane segments, four essential histidine residues involved in binding haem $b$, and a WWD domain that might play a crucial role during the formation of the covalent thioether bridges from haem $b$ and a suitable apocytochrome $c$.

The genetic strategies presented in this study offer the opportunity to attach a short affinity tag (His- or Streptag) to each of the W. succinogenes CCHL isoenzymes in order to attempt future purification by affinity chromatography. As CcsBA from $H$. hepaticus as well as B. subtilis ResB seem to be prone to proteolytic degradation in E. coli, it might be beneficial to use $W$. succinogenes as production host (Ahuja et al., 2009; Frawley \& Kranz, 2009). Although polytopic membrane-bound proteins or protein complexes cannot be easily purified, let alone crystallized, successful large-scale preparation of an intact CCHL will hopefully lead to a structural model in the future that might elucidate the many poorly understood molecular details of CCHL function.

\section{ACKNOWLEDGEMENTS}

This work was funded by the Deutsche Forschungsgemeinschaft (research grant SI 848/2-1 to J. S.). R. G. K. is supported by NIH grant GM 47909.

\section{REFERENCES}

Ahuja, U., Kjelgaard, P., Schulz, B. L., Thöny-Meyer, L. \& Hederstedt, L. (2009). Haem-delivery proteins in cytochrome $c$ maturation system II. Mol Microbiol 73, 1058-1071.

Barrick, D. (1994). Replacement of the proximal ligand of sperm whale myoglobin with free imidazole in the mutant His-93 $\rightarrow$ Gly. Biochemistry 33, 6546-6554.

Bode, C., Goebell, H. \& Stähler, E. (1968). Zur Eliminierung von Trübungsfehlern bei der Eiweißbestimmung mit der Biuretmethode. Z Klin Chem Klin Biochem 6, 418-422.

Dreyfuss, B. W., Hamel, P. P., Nakamoto, S. S. \& Merchant, S. (2003). Functional analysis of a divergent system II protein, Ccs1, involved in c-type cytochrome biogenesis. J Biol Chem 278, 26042613.

Feissner, R. E., Richard-Fogal, C. L., Frawley, E. R., Loughman, J. A., Earley, K. W. \& Kranz, R. G. (2006). Recombinant cytochromes $c$ biogenesis systems I and II and analysis of haem delivery pathways in Escherichia coli. Mol Microbiol 60, 563-577.

Ferguson, S. J., Stevens, J. M., Allen, J. W. A. \& Robertson, I. B. (2008). Cytochrome $c$ assembly: a tale of ever increasing variation and mystery? Biochim Biophys Acta 1777, 980-984.

Frawley, E. R. \& Kranz, R. G. (2009). CcsBA is a cytochrome $c$ synthetase that also functions in heme transport. Proc Natl Acad Sci U S A 106, 10201-10206.

Goddard, A. D., Stevens, J. M., Rondelet, A., Nomerotskaia, E., Allen, J. W. A. \& Ferguson, S. J. (2010). Comparing the substrate specificities of cytochrome $c$ biogenesis systems I and II: bioenergetics. FEBS J 277, 726-737. 
Hamel, P. P., Dreyfuss, B. W., Xie, Z., Gabilly, S. T. \& Merchant, S. (2003). Essential histidine and tryptophan residues in CcsA, a system II polytopic cytochrome $c$ biogenesis protein. J Biol Chem 278, 2593 2603.

Hartshorne, S., Richardson, D. J. \& Simon, J. (2006). Multiple haem lyase genes indicate substrate specificity in cytochrome $c$ biogenesis. Biochem Soc Trans 34, 146-149.

Hartshorne, R. S., Kern, M., Meyer, B., Clarke, T. A., Karas, M., Richardson, D. J. \& Simon, J. (2007). A dedicated haem lyase is required for the maturation of a novel bacterial cytochrome $c$ with unconventional covalent haem binding. Mol Microbiol 64, 1049-1060.

Kern, M. \& Simon, J. (2008). Characterization of the NapGH quinol dehydrogenase complex involved in Wolinella succinogenes nitrate respiration. Mol Microbiol 69, 1137-1152.

Kern, M. \& Simon, J. (2009a). Electron transport chains and bioenergetics of respiratory nitrogen metabolism in Wolinella succinogenes and other Epsilonproteobacteria. Biochim Biophys Acta 1787, 646-656.

Kern, M. \& Simon, J. (2009b). Periplasmic nitrate reduction in Wolinella succinogenes: cytoplasmic NapF facilitates NapA maturation and requires the menaquinol dehydrogenase $\mathrm{NapH}$ for membrane attachment. Microbiology 155, 2784-2794.

Kern, M., Mager, A. M. \& Simon, J. (2007). Role of individual nap gene cluster products in NapC-independent nitrate respiration of Wolinella succinogenes. Microbiology 153, 3739-3747.

Kern, M., Eisel, F., Scheithauer, J., Kranz, R. G. \& Simon, J. (2010). Substrate specificity of three cytochrome $c$ haem lyase isoenzymes from Wolinella succinogenes: unconventional haem $c$ binding motifs are not sufficient for haem $c$ attachment by Nrfl and CcsA1. Mol Microbiol 75, 122-137.

Kranz, R. G., Richard-Fogal, C., Taylor, J.-S. \& Frawley, E. R. (2009). Cytochrome $c$ biogenesis: mechanisms for covalent modifications and trafficking of heme and for heme-iron redox control. Microbiol Mol Biol Rev 73, 510-528.

Kröger, A., Geisler, V. \& Duchêne, A. (1994). Isolation of Wolinella succinogenes hydrogenase, chromatofocusing. In A Practical Guide to Membrane Protein Purification, pp. 141-147. Edited by G. von Jagow \& H. Schägger. London: Academic Press.

Merchant, S. S. (2009). His protects heme as it crosses the membrane. Proc Natl Acad Sci U S A 106, 10069-10070.

Pisa, R., Stein, T., Eichler, R., Gross, R. \& Simon, J. (2002). The nrfI gene is essential for the attachment of the active site haem group of Wolinella succinogenes cytochrome $c$ nitrite reductase. Mol Microbiol 43, 763-770.

Ren, Q., Ahuja, U. \& Thöny-Meyer, L. (2002). A bacterial cytochrome $c$ heme lyase. CcmF forms a complex with the heme chaperone CcmE and CcmH but not with apocytochrome $c$. J Biol Chem 277, 76577663.

Richard-Fogal, C. L. \& Kranz, R. G. (2010). The CcmC: heme : CcmE complex in heme trafficking and cytochrome $c$ biosynthesis. $J \mathrm{Mol}$ Biol 401, 350-362.

Richard-Fogal, C. L., Frawley, E. R., Feissner, R. E. \& Kranz, R. G. (2007). Heme concentration dependence and metalloprotein inhibition of the system I and II cytochrome $c$ assembly pathways. J Bacteriol 189, 455-463.

Richard-Fogal, C. L., Bonner, E. R., Zhu, H., San Francisco, B. \& Kranz, R. G. (2009). A conserved haem redox and trafficking pathway for cofactor attachment. EMBO J 28, 2349-2359.

Sambrook, J., Fritsch, E. F. \& Maniatis, T. (1989). Molecular Cloning: a Laboratory Manual, 2nd edn. Cold Spring Harbor, NY: Cold Spring Harbor Laboratory.

Sanders, C., Turkarslan, S., Lee, D.-W. \& Daldal, F. (2010). Cytochrome $c$ biogenesis: the Ccm system. Trends Microbiol 18, 266-274.

Schulz, H., Pellicioli, E. C. \& Thöny-Meyer, L. (2000). New insights into the role of $\mathrm{CcmC}, \mathrm{CcmD}$ and $\mathrm{CcmE}$ in the haem delivery pathway during cytochrome $c$ maturation by a complete mutational analysis of the conserved tryptophan-rich motif of CcmC. Mol Microbiol 37, 1379-1388.

Simon, J. (2002). Enzymology and bioenergetics of respiratory nitrite ammonification. FEMS Microbiol Rev 26, 285-309.

Simon, J. \& Kern, M. (2008). Quinone-reactive proteins devoid of haem $b$ form widespread membrane-bound electron transport modules in bacterial anaerobic respiration. Biochem Soc Trans 36, 1011-1016.

Simon, J., Gross, R., Ringel, M., Schmidt, E. \& Kröger, A. (1998). Deletion and site-directed mutagenesis of the Wolinella succinogenes fumarate reductase operon. Eur J Biochem 251, 418-426.

Simon, J., Gross, R., Einsle, O., Kroneck, P. M. H., Kröger, A. \& Klimmek, O. (2000). A NapC/NirT-type cytochrome $c(\mathrm{NrfH})$ is the mediator between the quinone pool and the cytochrome $c$ nitrite reductase of Wolinella succinogenes. Mol Microbiol 35, 686-696.

Simon, J., Pisa, R., Stein, T., Eichler, R., Klimmek, O. \& Gross, R. (2001). The tetraheme cytochrome $c \mathrm{NrfH}$ is required to anchor the cytochrome $c$ nitrite reductase (NrfA) in the membrane of Wolinella succinogenes. Eur J Biochem 268, 5776-5782.

Simon, J., van Spanning, R. J. M. \& Richardson, D. J. (2008). The organisation of proton motive and non-proton motive redox loops in prokaryotic respiratory systems. Biochim Biophys Acta 1777, 14801490.

Edited by: P. Cornelis 